International Journal of

Environmental Research and

Public Health

ISSN 1660-4601

www.mdpi.com/journal/ijerph

Communication

\title{
Geographic Elevation and Cognitive Function among Elderly Residents in Rural Mountainous Areas: Shimane CoHRE Study
}

\author{
Tsuyoshi Hamano ${ }^{1, *}$, Keiichi Onoda ${ }^{2}$, Miwako Takeda ${ }^{1}$, Kristina Sundquist ${ }^{3,4}$, \\ Shuhei Yamaguchi ${ }^{2}$ and Toru Nabika ${ }^{1,5}$
}

1 Center for Community-Based Health Research and Education (CoHRE), Organization for the Promotion of Project Research, Shimane University, 223-8 Enya-cho, Izumo, Shimane 693-8501, Japan; E-Mails: cohre1@med.shimane-u.ac.jp (M.T.); nabika@med.shimane-u.ac.jp (T.N.)

2 Department of Neurology, Shimane University School of Medicine, 89-1 Enya-cho, Izumo, Shimane 693-8501, Japan; E-Mails: onodak1@med.shimane-u.ac.jp (K.O.); yamagu3n@med.shimane-u.ac.jp (S.Y.)

3 Center for Primary Health Care Research, Lund University, Clinical Research Centre (CRC), Building 28, floor 11, Jan Waldenströms gata 35, Skåne University Hospital, SE-205 02 Malmö, Sweden; E-Mail: kristina.sundquist@med.lu.se

4 Stanford Prevention Research Center, Stanford University, Medical School Office Building (MSOB), 251 Campus Drive MC 5411, Stanford, CA 94305, USA

5 Department of Functional Pathology, Shimane University School of Medicine, 89-1 Enya-cho, Izumo, Shimane 693-8501, Japan

* Author to whom correspondence should be addressed; E-Mail: thamano@med.shimane-u.ac.jp; Tel.: +81-853-20-2918; Fax: +81-853-20-2586.

Academic Editor: Paul B. Tchounwou

Received: 16 September 2015 / Accepted: 21 October 2015 / Published: 23 October 2015

\begin{abstract}
The aim of this study was to test whether there is an association between elevation and cognitive function among elderly residents in rural mountainous areas. Data were collected in 2012 from a cross-sectional study conducted in Ohnan Town, which is located in a rural mountainous area in the southern part of Shimane Prefecture, Japan. Cognitive function was evaluated using CADi (Cognitive Assessment for Dementia, iPad version) and elevation was estimated by using Geographic Information Systems according to the participant's address. After excluding subjects with missing data, 866 participants were analyzed. After adjustment for potential confounding factors, higher elevation was significantly associated
\end{abstract}


with decreased cognitive function. This finding suggests that it is important to consider the physical environment, i.e., elevation, that would affect accessibility to health-promoting goods, services, and resources when seeking to maintain cognitive function in elderly people living in rural mountainous areas.

Keywords: cognitive function; elevation; rural mountainous area; elderly people

\section{Introduction}

Decreasing cognitive function is a major public health concern as the population ages [1]. Several risk factors for cognitive function have been postulated, including sociodemographic factors (e.g., age and education), lifestyle factors (e.g., smoking and diet), and health conditions (e.g., current history of a disease) [2-9]. More recently, research on the association between the physical environment, e.g., geographic elevation, and age-related diseases has gained momentum, with an increased focus on Geographic Information Systems (GIS) [10-15].

A previous study conducted in Japan found that people living at higher elevations were more likely to have decreased masticatory ability [12]. Additionally, people living at higher elevations had a higher salt intake than those living at lower elevations [10]. In Japan, hilly and mountainous areas occupy approximately $70 \%$ of the country and are defined as regions extending from the outer plains to the mountains [16]. Health-promoting goods, services, and resources, which may have a positive effect on cognitive function, are substantially limited at higher elevations as compared to lower elevations. It is therefore hypothesized that elderly people who live at higher elevations have lower cognitive function compared to those who live at lower elevations. To the best of our knowledge, no previous study has investigated the hypothesized associations.

The aim of this study was to test whether there is an association between elevation and cognitive function among elderly residents in rural mountainous areas. Cognitive function was evaluated using CADi (Cognitive Assessment for Dementia, iPad version), which can be run on a tablet computer for mass screening in the community [17].

\section{Methods}

\subsection{Study Design}

Data were collected from a cross-sectional study conducted in 2012. The present study was a part of the Shimane CoHRE Study, which was designed to examine the determinants of age-related diseases, including cognitive function [10-15,17]. The Shimane CoHRE study was conducted by Shimane University, Japan, in collaboration with a health examination program that involved Ohnan Town. This town is located in a rural mountainous area in the southern part of Shimane Prefecture, Japan.

Health examination programs are available once a year for individuals between 40 and 74 years of age who are covered by National Health Insurance. The residents in this town have two options when they wish to undergo a health examination: They may participate in a group examination conducted at public health centers, or they may receive an individual examination conducted at medical institutions. 
We were permitted to use data on group examinations for our analysis. After excluding participants with missing data (36 participants), we analyzed data from 866 participants. The study protocol was approved by the Ethics Committee of Shimane University School of Medicine in 2010. Written informed consent was obtained from all participants.

\subsection{Cognitive Function}

Cognitive function was assessed using CADi which has been evaluated on validity and reliability [17]. CADi consists of 10 separate items: immediate recognition of three words, question about semantic memory, categorization of six objects, subtraction, backward repetition of three digits, cube rotation, pyramid rotation, trail-making test $\mathrm{A}$, trail-making test $\mathrm{B}$, and delayed recognition of three words. The score ranges from 0 to 10 , with higher scores indicating better cognitive function. The participants answered during the voice presentation of a question statement using an iPad (https://itunes.apple.com/ us/app/cadi/id586052447, currently Japanese version only).

\subsection{Elevation}

Geographic Information Systems (ArcGIS software, version 10.0, Environmental Systems Research Institute, Redlands, CA, USA) was employed for database queries and used to estimate elevation based on the individuals' addresses. The elevation for each participant was assessed using the ArcGIS ready-to-use dataset of digital elevation models (mean value $=252.8 \mathrm{~m}$; range, $72 \mathrm{~m}$ to $465 \mathrm{~m}$ ).

\subsection{Other Measures}

We considered the following variables in the analysis: age (years, analyzed as a continuous variable), gender (male vs. female), body mass index (BMI) $\left(\mathrm{kg} / \mathrm{m}^{2}\right.$, analyzed as a continuous variable), current smoker (yes vs. no), current alcohol drinker (yes vs. no), physically active (engaged in physical activity regularly $=$ yes $v s$. not engaged in physical activity regularly $=$ no), use of medication for disease treatment (medication to reduce blood pressure, medication to reduce blood sugar or insulin injections, and medication to reduce cholesterol level), accessible transportation (driver $=$ yes $v s$. non-driver $=$ no $)$, and a self-rating depression scale (SDS) (analyzed as a continuous variable).

\subsection{Statistical Analysis}

Descriptive statistics were calculated. Multivariable linear regression models were performed to derive regression coefficients (B), standard errors (SE), and $p$-values. Independent variables were coded as follows: Gender $(0=$ male, $1=$ female $)$, and current smoker, current alcohol drinker, regular physical activity, medication for disease treatment, and accessible transportation $(0=$ no, $1=$ yes $)$. $P$-values less than 0.05 were considered statistically significant. All statistical analyses were performed using IBM SPSS Statistics 20 (IBM Corporation, Tokyo, Japan). 


\section{Results}

The characteristics of the study participants are shown in Table 1 . The study consisted of 866 participants (41.2\% male and 58.8\% female) and the mean (standard deviation: SD) age was 65.8 (7.7) years. The mean (SD) CADi score was 8.1 (1.5). Of the participants, 110 (12.7\%) were smokers, and $461(53.2 \%)$ reported drinking alcohol. Around $30 \%$ of participants were physically active; 303 participants $(35.0 \%)$ took medication for hypertension and 664 participants $(76.7 \%)$ were drivers. The mean (SD) BMI and SDS (SD) were $22.7(3.1) \mathrm{kg} / \mathrm{m}^{2}$ and $35.0(8.2)$, respectively.

Table 1. Characteristics of the study participants.

\begin{tabular}{ccc}
\hline Variables & $\begin{array}{c}\text { Number of } \\
\text { Participants }\end{array}$ & \% or Mean (SD) \\
\hline CADi score, mean (SD) & 866 & $8.1(1.5)$ \\
Elevation, m (SD) & 866 & $252.8(74.5)$ \\
Age, years (SD) & 866 & $65.8(7.7)$ \\
Gender (male), \% & 357 & 41.2 \\
Current smoker, \% & 110 & 12.7 \\
Current alcohol drinker, \% & 461 & 53.2 \\
Regular physical activity, \% & 280 & 32.3 \\
Medication & & \\
To reduce blood pressure, \% & 303 & 35.0 \\
To reduce blood sugar or insulin injections, \% & 55 & 6.4 \\
To reduce cholesterol level, \% & 191 & 22.1 \\
Body Mass Index, kg/m ${ }^{2}$ & 866 & $22.7(3.1)$ \\
Driver, \% & 664 & 76.7 \\
SDS, mean (SD) & 866 & $35.0(8.2)$ \\
\hline
\end{tabular}

CADi, Cognitive Assessment for Dementia, iPad version; SD, standard deviation; SDS, self-rating depression scale.

Table 2 shows the results of the multivariable liner regression analysis. Higher elevation was significantly associated with decreased cognitive function (regression coefficient $=-0.002, p=0.016$ ). Age, gender, medication for hypertension, and being a driver were also significantly associated with cognitive function (regression coefficient $=-0.044, p<0.001$; regression coefficient $=0.345$, $p=0.004$; regression coefficient $=-0.313, p=0.007$; regression coefficient $=0.718$, $p<0.001$, respectively).

\section{Discussion}

To the best of our knowledge, no study has examined the association between elevation and cognitive function among elderly people. As we hypothesized, our results showed that higher elevation is significantly associated with decreased cognitive function. These results suggest that elevation could be an important determinant of cognitive function in rural mountainous areas. 
Table 2. Multivariable linear regression analysis with cognitive function as dependent variable.

\begin{tabular}{ccccc}
\hline Variables & B & SE & $\boldsymbol{t}$ & $\boldsymbol{p}$-Value \\
\hline Age & -0.044 & 0.007 & -6.367 & $<0.001$ \\
Gender & 0.345 & 0.119 & 2.894 & 0.004 \\
Current smoker & 0.220 & 0.162 & 1.360 & 0.174 \\
Current alcohol drinker & 0.037 & 0.109 & 0.335 & 0.738 \\
Regular physical activity & -0.055 & 0.108 & -0.506 & 0.613 \\
Medication & & & & \\
To reduce blood pressure & -0.313 & 0.115 & -2.711 & 0.007 \\
To reduce blood sugar or insulin injections & 0.028 & 0.210 & 0.132 & 0.895 \\
To reduce cholesterol level & 0.211 & 0.129 & 1.633 & 0.103 \\
Body Mass Index & -0.028 & 0.017 & -1.721 & 0.086 \\
Driver & 0.718 & 0.126 & 5.693 & $<0.001$ \\
SDS & -0.008 & 0.006 & -1.324 & 0.186 \\
Elevation & -0.002 & 0.001 & -2.419 & 0.016 \\
\hline
\end{tabular}

SDS, self-rating depression scale.

This study advances the recent debate on the association between the physical environment, i.e., elevation, and health. As noted, a previous study found that people living at higher elevations are more likely to have decreased masticatory ability [12]. Also, overweight individuals living at a high elevation had a higher risk of chronic knee pain than non-overweight individuals living at a low elevation [13]. The mechanisms underlying the association between elevation and health outcomes are most likely of a complex nature, and causal inferences remain to be established. Considering that our participants live in a rural mountainous area, people living at higher elevations may be more exposed to the effects of relatively limited access to health-promoting goods, services, and resources than those living at lower elevations. For example, previous research conducted in rural mountainous areas found that people living at higher elevations had a higher salt intake than those living at lower elevations [10]. Further studies are required to examine whether health behaviors differ by the elevation in rural mountainous areas.

Decreasing cognitive function is a major public health concern as the population ages [1]. Health professionals should manage efficient health plans that can select appropriate target groups for interventions. Our results suggest that people living at higher elevations should be considered a priority target to measure cognitive function. CADi, which was used in this study, is a useful instrument for mass screening in the community due to its low cost, brief administration time, and user-friendliness for elderly people [17], so understanding the physical environment, i.e., elevation, and using CADi can help health professionals promote efficient activities in the community.

There are a number of potential limitations in the current study. First, our data set from group examination may not be an entirely representative sample. A selection bias caused by non-respondents was present and may have influenced the associations shown in our results. Caution is therefore warranted in over-interpreting this finding. However, the participation rate of the group examination $(37.1 \%)$ was higher than that of the individual examination (19.1\%) and $43.8 \%$ of residents did not receive health examinations during this year. Second, misclassification may have occurred in the self-reported data as a consequence of recall errors. However, we have no reason to believe that this potential bias differed between the residential areas. Third, our data did not allow for the assessment of other important 
confounding factors such as education and income. Finally, the present study used a cross-sectional design that did not allow us to establish temporal ordering or causal relationships.

\section{Conclusions}

Our analysis indicated that high elevation was significantly associated with decreased cognitive function. This finding suggests that it is important to consider the physical environment, i.e., elevation, when seeking to maintain cognitive function in the elderly living in rural mountainous areas because this may affect their accessibility to health-promoting goods, services, and resources. Longitudinal research is needed to confirm these findings and explore the underlying mechanisms.

\section{Acknowledgments}

This work was supported by MEXT KAKENHI Grant Number $15 \mathrm{H} 05365$.

\section{Author Contributions}

Tsuyoshi Hamano, Kristina Sundquist, Shuhei Yamaguchi, and Toru Nabika contributed to the planning of the study design and interpretation of the data. Tsuyoshi Hamano, Keiichi Onoda, and Miwako Takeda collected the data. Tsuyoshi Hamano and Miwako Takeda performed the data analysis. All authors approved the final version of the manuscript.

\section{Conflicts of Interest}

The authors declare no conflict of interest. The founding sponsors had no role in the design of the study; in the collection, analyses, or interpretation of data; in the writing of the manuscript, and in the decision to publish the results.

\section{References}

1. Ferri, C.P.; Prince, M.; Brayne, C.; Brodaty, H.; Fratiglioni, L.; Ganguli, M.; Hall, K.; Hasegawa, K.; Hendrie, H.; Huang, Y.; et al. Global prevalence of dementia: A Delphi consensus study. Lancet 2005, 366, 2112-2117.

2. Goate, A.; Chartier-Harlin, M.C.; Mullan, M.; Brown, J.; Crawford, F.; Fidani, L.; Giuffra, L.; Haynes, A.; Irving, N.; James, L.; et al. Segregation of a missense mutation in the amyloid precursor protein gene with familial Alzheimer's disease. Nature 1991, 349, 704-706.

3. Sherrington, R.; Rogaev, E.I.; Liang, Y.; Rogaeva, E.A.; Levesque, G.; Ikeda, M.; Chi, H.; Lin, C.; Li, G.; Holman, K.; et al. Cloning of a gene bearing missense mutations in early-onset familial Alzheimer's disease. Nature 1995, 375, 754-760.

4. Morris, M.C.; Evans, D.A.; Tangney, C.C.; Bienias, J.L.; Wilson, R.S. Associations of vegetable and fruit consumption with age-related cognitive change. Neurology 2006, 67, 1370-1376.

5. Almeida, O.P.; Hulse, G.K.; Lawrence, D.; Flicker, L. Smoking as a risk factor for Alzheimer's disease: Contrasting evidence from a systematic review of case-control and cohort studies. Addiction 2002, 97, 15-28. 
6. Shumaker, S.A.; Legault, C.; Kuller, L.; Rapp, S.R.; Thal, L.; Lane, D.S.; Fillit, H.; Stefanick, M.L.; Hendrix, S.L.; Lewis, C.E.; et al. Conjugated equine estrogens and incidence of probable dementia and mild cognitive impairment in postmenopausal women: Women's health initiative memory study. J. Am. Med. Assco. 2004, 291, 2947-2958.

7. Forette, F.; Seux, M.L.; Staessen, J.A.; Thijs, L.; Babarskiene, M.R.; Babeanu, S.; Bossini, A.; Fagard, R.; Gil-Extremera, B.; Laks, T.; et al. The prevention of dementia with antihypertensive treatment: New evidence from the Systolic Hypertension in Europe (Syst-Eur) study. Arch. Int. Med. 2002, 162, 2046-2052.

8. Qiu, C.; von Strauss, E.; Fastbom, J.; Winblad, B.; Fratiglioni, L. Low blood pressure and risk of dementia in the Kungsholmen project: A 6-year follow-up study. Arch. Neurol. 2003, 60, 223-228.

9. Lee, S.; Kawachi, I.; Berkman, L.F.; Grodstein, F. Education, other socioeconomic indicators, and cognitive function. Am. J. Epidemiol. 2003, 157, 712-720.

10. Ferdaus, S.I.; Kohno, K.; Hamano, T.; Takeda, M.; Yamasaki, M.; Isomura, M.; Shiwaku, K.; Nabika, T. Altitudes of residential areas affect salt intake in a rural area in Japan: A Shimane CoHRE Study. Hypertens. Res. 2015, doi:10.1038/hr.2015.91.

11. Takeda, M.; Hamano, T.; Kohno, K.; Yano, S.; Shiwaku, K.; Nabika, T. Association between geographic elevation, bone status, and exercise habits: The Shimane CoHRE Study. Int. J. Environ. Res. Public Health 2015, 12, 7392-7399.

12. Hamano, T.; Tominaga, K.; Takeda, M.; Sundquist, K.; Nabika, T. Accessible transportation, geographic elevation, and masticatory ability among elderly residents of a rural area. Int. J. Environ. Res. Public Health 2015, 12, 7199-7207.

13. Hamano, T.; Kamada, M.; Kitayuguchi, J.; Sundquist, K.; Sundquist, J.; Shiwaku, K. Association of overweight and elevation with chronic knee and low back pain: A cross-sectional study. Int. J. Environ. Res. Public Health 2014, 11, 4417-4426.

14. Hamano, T.; Kimura, Y.; Takeda, M.; Yamasaki, M.; Isomura, M.; Nabika, T.; Shiwaku, K. Effect of environmental and lifestyle factors on hypertension: Shimane COHRE study. PLoS ONE 2012, 7, doi:10.1371/journal.pone.0049122.

15. Hamano, T.; Kimura, Y.; Takeda, M.; Yamasaki, M.; Nabika, T.; Shiwaku, K. Is location associated with high risk of hypertension? Shimane COHRE Study. Am. J. Hypertens. 2012, 25, 784-788.

16. Ueyama, H. Estimating hourly direct and diffuse solar radiation for the compilation of solar radiation distribution maps. J. Agric. Meteor. 2005, 61, 207-216.

17. Onoda, K.; Hamano, T.; Nabika, Y.; Aoyama, A.; Takayoshi, H.; Nakagawa, T.; Ishihara, M.; Mitaki, S.; Yamaguchi, T.; Oguro, H.; et al. Validation of a new mass screening tool for cognitive impairment: Cognitive assessment for dementia, iPad version. Clin. Interv. Aging 2013, 8, 353-360.

(C) 2015 by the authors; licensee MDPI, Basel, Switzerland. This article is an open access article distributed under the terms and conditions of the Creative Commons Attribution license (http://creativecommons.org/licenses/by/4.0/). 